Research Article

\title{
General Minimum Lower-Order Confounding Designs with Multi-Block Variables
}

\author{
Yuna Zhao \\ School of Mathematics and Statistics, Shandong Normal University, Jinan 250358, China \\ Correspondence should be addressed to Yuna Zhao; yunazhao0504@163.com
}

Received 7 February 2021; Revised 31 March 2021; Accepted 8 April 2021; Published 21 April 2021

Academic Editor: Gengxin Sun

Copyright (@) 2021 Yuna Zhao. This is an open access article distributed under the Creative Commons Attribution License, which permits unrestricted use, distribution, and reproduction in any medium, provided the original work is properly cited.

Blocking the inhomogeneous units of experiments into groups is an efficient way to reduce the influence of systematic sources on the estimations of treatment effects. In practice, there are two types of blocking problems. One considers only a single block variable and the other considers multi-block variables. The present paper considers the blocking problem of multi-block variables. Theoretical results and systematical construction methods of optimal blocked $2^{n-m}$ designs with $(N / 4)+1 \leq n \leq 5 N / 16$ are developed under the prevalent general minimum lower-order confounding (GMC) criterion, where $N=2^{n-m}$.

\section{Introduction}

The regular $2^{n-m}$ factorial experiment has played an important role in engineering, manufacturing industry, agriculture and medicine, and so on. It allows efficient and economic experimentation to estimate treatment effects. When the size of the experimental units is large, the inhomogeneity will cause unwanted variance to the estimations of treatment effects. To reduce such bad influence, a crucial way is to partition the experimental units into blocks.

There are two kinds of blocking problems as pointed out in [1]. One is called the single block variable problem which considers only a single block variable, and the other is called the multi-block variable problem which considers two or more block variables. In the last decades, choosing optimal blocked $2^{n-m}$ designs with a single block variable has been well investigated; for example, the authors in [2-9] studied the blocked $2^{n-m}$ designs under different minimum aberration criteria; Chen et al. and Zhao et al. [10,11] explored the blocked $2^{n-m}$ designs under the clear effects criterion; Zhang and Mukerjee, Zhao et al., and Zhao and Zhao [12-14] explored the constructions of the blocked $2^{n-m}$ designs under the general minimum lower-order confounding (GMC) criterion proposed in [15]; and Zhao et al.
[16-18] gave construction methods of the blocked $2^{n-m}$ designs under another GMC criterion proposed in [19].

Compared to the large body of work on the blocking problem of single block variable, the studies on choosing optimal blocked $2^{n-m}$ designs with multi-block variables are relatively rare. However, it has been recognized that the blocking problem of multi-block variables can arise quite naturally in many practical situations. For example, in the agricultural context, Bisgaard [1] pointed that when designs are laid out in rectangular schemes, both row and column inhomogeneity effects probably exist in the soil. Another example of multi-block variables is from [20]. Considering the comparison of two gasoline additives by testing them on two cars with two drivers over two days, the "cars," "drivers," and "days" are three block variables which should be taken into account when performing experiments.

Under the clear effects criterion, Zhao and Zhao [21] proposed an algorithm for finding optimal blocked $2^{n-m}$ designs with multi-block variables. Under the minimum aberration criterion, Zhao and Zhao [22] developed some rules for constructing optimal blocked $2^{n-m}$ designs with multi-block variables. Zhang et al. [23] extended the idea of GMC criterion to the case of multi-block variable problem and developed the blocked GMC criterion, called 
the $B^{2}$-GMC criterion. Inheriting the advantage of the GMC criterion, the $B^{2}$-GMC designs are particularly preferable when some prior information on the importance ordering of treatment effects is present. By computer search, Zhang et al. [23] tabulated some $B^{2}$-GMC deigns with small $n$ and $N$, where $N=2^{n-m}$. When $n$ or $N$ is large, computer search becomes computationally expensive. Zhao et al. [24] and Zhao and Zhao [25] completed the constructions of $B^{2}$-GMC designs with $5 N / 16+1 \leq n \leq N-1$. This paper aims at providing theories and systematical construction methods of the $B^{2}$-GMC designs with $N / 4+1 \leq n \leq 5 N / 16$.

The rest of the paper is organized as follows. Section 2 reviews doubling theory and $B^{2}$-GMC criterion. Section 3 provides theoretical results and construction methods of $B^{2}$-GMC designs. Section 4 gives concluding remarks. Some useful lemmas are deferred to Appendix.

\section{Preliminaries}

2.1. Doubling Theory. Let $\mathbf{X}=\left(\mathbf{x}_{1}, \ldots, \mathbf{x}_{t}\right)$ be a matrix with entries 1 or -1 . Denote $\mathbf{J}_{0}=(1,1)^{\prime}$ and $\mathbf{J}_{1}=(1,-1)^{\prime}$, where ${ }^{\prime}$ denotes transpose. Define

$$
D(\mathbf{X})=\left(\left(\mathbf{J}_{0}, \mathbf{J}_{1}\right) \otimes \mathbf{x}_{1}, \ldots,\left(\mathbf{J}_{0}, \mathbf{J}_{1}\right) \otimes \mathbf{x}_{t}\right),
$$

as a double of $\mathbf{X}$, where $\otimes$ is the Kronecker product. Let $D^{q}(\mathbf{X})$ denote the design obtained by repeatedly doubling $\mathbf{X} q$ times, i.e., $D^{q}(\mathbf{X})=D\left(D^{q-1}(\mathbf{X})\right)$. When $\mathbf{X}=1$, we write $D^{q}(1)=\left(\mathbf{I}_{2 q}, \mathbf{1}_{2 q}, \mathbf{2}_{2^{q}}, \mathbf{1}_{2^{q}} \mathbf{2}_{2^{q}}, \ldots, \mathbf{1}_{2^{q}} \mathbf{2}_{2^{q}} \mathbf{3}_{2^{q}}, \ldots, \mathbf{q}_{2^{q}}\right)$, where the subscript $2^{q}$ means the dimension of a column, $\mathbf{I}_{2^{q}}$ is a column of 1 's,

$$
\begin{aligned}
\mathbf{1}_{2^{q}}^{\prime} & =(1, \ldots, 1,-1, \ldots,-1) \\
\mathbf{2}_{2^{q}}^{\prime} & =(1, \ldots, 1,-1, \ldots,-1, \ldots, 1, \ldots, 1,-1, \ldots,-1) \\
& \vdots \\
\mathbf{q}_{2^{q}}^{\prime} & =(1,-1,1,-1, \ldots, 1,-1,1,-1),
\end{aligned}
$$

are $q$ independent columns, and the other columns are the component-wise products of some of these $q$ independent columns. For example, $\mathbf{1}_{2 q} \mathbf{2}_{2 q}$ is the component-wise product of the columns $\mathbf{1}_{2^{q}}$ and $\boldsymbol{2}_{2^{q}}$. Write $D^{q}(1)=$ $\left(\mathbf{I}_{2^{q}}, D^{q}(\cdot)\right)$; then, $D^{q}(\cdot)$ is just the regular two-level saturated design with columns arranged in Yates order. As some subdesigns in $D^{q}(\cdot)$, denote $\mathbf{H}_{0}^{q}=\varnothing, \quad \mathbf{H}_{1}^{q}=\mathbf{1}_{2 q}, \mathbf{H}_{r}^{q}=$ $\left(\mathbf{H}_{r-1}^{q}, \mathbf{r}_{2 q}, \mathbf{r}_{2^{q}} \mathbf{H}_{r-1}^{q}\right)$ for $r=2,3, \ldots, q$, where $\varnothing$ denotes the empty set, the superscript $q$ of $\mathbf{H}_{r}^{q}$ refers to that $\mathbf{H}_{r}^{q}$ is a subdesign of $D^{q}(\cdot)$, and $\mathbf{r}_{2 q} \mathbf{H}_{r-1}^{q}=\left(\mathbf{r}_{2 q} \mathbf{d}_{2 q}: \mathbf{d}_{2 q} \in \mathbf{H}_{r-1}^{q}\right)$, i.e., $\mathbf{H}_{r}^{q}$ consists of the first $2^{r}-1$ columns of $D^{q}(\cdot)$. Especially, $\mathbf{H}_{q}^{q}=D^{q}(\cdot)$.

2.2. $B^{2}-G M C$ Criterion. Before introducing the $B^{2}$-GMC criterion, we first review some principles in the multi-block variable problem. Let $b_{1}, b_{2}, \ldots, b_{s}$ denote the $s$ block variables which cause the inhomogeneity of the experimental units. Suppose that the block variable $b_{j}$ partitions the $N$ (= $2^{n-m}$ ) experimental units into $2^{l_{j}}$ blocks; then, $l_{j}$ independent columns are needed to carry out this blocking plan.
Denote $S_{j}$ as the set of the $l_{j}$ independent columns related to the block variable $b_{j}$. The block columns should follow the following rules:

(i) The $l_{j}$ block columns in $S_{j}(j=1,2, \ldots, s)$ are independent of each other.

(ii) A block column from $S_{j}$ is not necessarily independent of the block columns from $S_{i}$ with $j \neq i$.

In this paper, we focus on the case where each block variable is at two levels, i.e., $l_{j}=1$.

The effect hierarchy principle for blocked designs with multi-block variables is as follows (see [23]):

(i) The lower-order treatment factorial effects are more likely to be important than the higher-order ones, and the treatment factorial effects of the same order are equally likely to be important.

(ii) The lower-order block factorial effects are more likely to be important than the higher-order ones, and the block factorial effects of the same order are equally likely to be important.

(iii) All the interactions between treatment factors and block factors are negligible.

Since each variable or factor is assigned to one column of the design matrix when an experiment is carried out, we do not differentiate the variable, factor, and column. Based on the effect hierarchy principle and weak assumption that the effects involving three or more factors are usually not important and negligible, Zhang et al. [23] proposed the $B^{2}$-GMC criterion which pays attention to only the confounding among main treatment effects and the two-factor interactions of treatment factors (2fi's for short). For the same reason, a common assumption in blocking problem is that only the main effects of block variables and the interactions of any two block factors are potentially significant, and if a treatment effect is confounded with a potentially significant block effect, the treatment effect cannot be estimated. Thus, the confounding between the main effects of treatment factors and any potentially significant block effect is not allowed.

Denote $\mathbf{D}=\left(\mathbf{D}_{t}: \mathbf{D}_{b}\right)$ as a $2^{n-m}: 2^{s}$ design, where $\mathbf{D}_{t}$ consists of $n$ treatment factors corresponding to a regular $2^{n-m}$ design and $\mathbf{D}_{b}$ consists of $s$ block factors each of which can partition the $2^{n-m}$ runs into 2 blocks. Denote ${ }_{1}^{\#} C_{2}^{(p)}(\mathbf{D})$ as the number of main treatment effects which are aliased with $p 2$ fi's but not with any potentially significant block effects, where $p=0,1,2, \ldots, P$ and $P=n(n-1) / 2$. Similarly, ${ }_{2}^{\#} C_{2}^{(p)}(\mathrm{D})$ denotes the number of 2 fi's which are aliased with the other $p 2$ fi's but not with any potentially significant block effects, where $p=0,1, \ldots, P$. Denote

$$
\begin{aligned}
& { }_{1}^{\#} C_{2}(\mathbf{D})=\left({ }_{1}^{\#} C_{2}^{(0)}(\mathbf{D}),{ }_{1}^{\#} C_{2}^{(1)}(\mathbf{D}), \ldots,{ }_{1}^{\#} C_{2}^{(p)}(\mathbf{D})\right), \\
& { }_{2}^{\#} C_{2}(\mathbf{D})=\left({ }_{2}^{\#} C_{2}^{(0)}(\mathbf{D}),{ }_{2}^{\#} C_{2}^{(1)}(\mathbf{D}), \ldots,{ }_{2}^{\#} C_{2}^{(p)}(\mathbf{D})\right), \\
& { }^{\#} C(\mathbf{D})=\left({ }_{1}^{\#} C_{2}(\mathbf{D}),{ }_{2}^{\#} C_{2}(\mathbf{D})\right) .
\end{aligned}
$$


A $2^{n-m}: 2^{s}$ blocked design $\mathbf{D}=\left(\mathbf{D}_{t}: \mathbf{D}_{b}\right)$ is called a $B^{2}$-GMC design if it sequentially maximizes (4). Let ${ }_{1}^{\#} C_{2}^{(p)}\left(\mathbf{D}_{t}\right)$ be the number of main effects which are aliased with $p 2$ fi's of $\mathbf{D}_{t}$ and ${ }_{2}^{\#} C_{2}^{(p)}\left(\mathbf{D}_{t}\right)$ be the number of 2 fi's which are aliased with the other $p$ 2fi's of $\mathbf{D}_{t}$. Let

$$
\begin{aligned}
& { }_{1}^{\#} C_{2}\left(\mathbf{D}_{t}\right)=\left({ }_{1}^{\#} C_{2}^{(0)}\left(\mathbf{D}_{t}\right),{ }_{1}^{\#} C_{2}^{(1)}\left(\mathbf{D}_{t}\right), \ldots,{ }_{1}^{\#} C_{2}^{(p)}\left(\mathbf{D}_{t}\right)\right), \\
& { }_{2}^{\#} C_{2}\left(\mathbf{D}_{t}\right)=\left({ }_{2}^{\#} C_{2}^{(0)}\left(\mathbf{D}_{t}\right),{ }_{2}^{\#} C_{2}^{(1)}\left(\mathbf{D}_{t}\right), \ldots,{ }_{2}^{\#} C_{2}^{(p)}\left(\mathbf{D}_{t}\right)\right), \\
& { }^{\#} C\left(\mathbf{D}_{t}\right)=\left({ }_{1}^{\#} C_{2}\left(\mathbf{D}_{t}\right),{ }_{2}^{\#} C_{2}\left(\mathbf{D}_{t}\right)\right) .
\end{aligned}
$$

A $2^{n-m}$ design $\mathbf{D}_{t}$ is called a GMC design if $\mathbf{D}_{t}$ sequentially maximizes (5).

Let $q=n-m$. Constructing a $B^{2}$-GMC design is to choose $\mathbf{D}_{t}$ and $\mathbf{D}_{b}$ from $D^{q}(\cdot)$ such that (4) is sequentially maximized. In the following, without causing confusions, we omit the subscript of a column and the superscript of a design when they are taken from $D^{q}(\cdot)$. For example, we use $\mathbf{a}, \mathbf{H}_{r}$, and $\mathbf{H}_{q}$ instead of $\mathbf{a}_{2^{q}}, \mathbf{H}_{r}^{q}$, and $\mathbf{H}_{q}^{q}$, respectively. Denote

$$
U\left(\mathbf{D}_{b}\right)=\left\{\gamma \in \mathbf{H}_{q}: \gamma \in \mathbf{D}_{b} \text { or } \gamma=\mathbf{a b} \text { with } \mathbf{a}, \mathbf{b} \in \mathbf{D}_{b}\right\},
$$

and then $U\left(\mathbf{D}_{b}\right)$ consists of all the potentially significant block effects. As previously stated, the confounding between main treatment effects and potentially significant block effects is not allowed. This requires $\mathbf{D}_{t} \cap U\left(\mathbf{D}_{b}\right)=\varnothing$, and thus ${ }_{1}^{\#} C_{2}^{(p)}(\mathbf{D})={ }_{1}^{\#} C_{2}^{(p)}\left(\mathbf{D}_{t}\right)$ for $p=0,1,2, \ldots, P$.

For $\mathbf{D}_{t} \subset \mathbf{H}_{q}$ and $\boldsymbol{\gamma} \in \mathbf{H}_{q}$, define

$$
B_{2}\left(\mathbf{D}_{t}, \boldsymbol{\gamma}\right)=\#\left\{\left(\mathbf{d}_{1}, \mathbf{d}_{2}\right): \mathbf{d}_{1}, \mathbf{d}_{2} \in \mathbf{D}_{t}, \mathbf{d}_{1} \mathbf{d}_{2}=\boldsymbol{\gamma}\right\},
$$

where \# denotes the cardinality of a set and $\mathbf{d}_{1} \mathbf{d}_{2}$ stands for the two-factor interaction of $\mathbf{d}_{1}$ and $\mathbf{d}_{2}$. Thus, $B_{2}\left(\mathbf{D}_{t}, \gamma\right)$ is the number of 2fi's of $\mathbf{D}_{t}$ appearing in the alias set that contains $\gamma$.

Isomorphism introduced by Tang and $\mathrm{Wu}$ [26] is a useful concept which helps narrow down the search of the optimal blocked designs here. An isomorphism $\phi$ is a one-toone mapping from $\mathbf{H}_{q}$ to $\mathbf{H}_{q}$ such that $\phi(\mathbf{x y})=\phi(\mathbf{x}) \phi(\mathbf{y})$ for every $\mathbf{x} \neq \mathbf{y} \in \mathbf{H}_{q}$. The $2^{n-m}$ designs $\mathbf{D}_{t}$ and $\mathbf{D}_{t}^{*}$ are isomorphic if there exists an isomorphism $\phi$ that maps $\mathbf{D}_{t}$ onto $\mathbf{D}_{t}^{*}$. The $2^{n-m}: 2^{s}$ designs $\mathbf{D}=\left(\mathbf{D}_{t}: \mathbf{D}_{b}\right)$ and $\mathbf{D}^{*}=\left(\mathbf{D}_{t}^{*}: \mathbf{D}_{b}^{*}\right)$ are isomorphic if there exists an isomorphism $\phi$ that maps $\mathbf{D}_{t}$ onto $\mathbf{D}_{t}^{*}$ and $\mathbf{D}_{b}$ onto $\mathbf{D}_{b}^{*}$.

\section{Constructions of $B^{2}$-GMC Designs}

3.1. $B^{2}-G M C 2^{n-m}: 2^{s}$ Designs with $n=(N / 4)+1$. A design is of MaxC2 (see [27]) if it has resolution IV and maximum number of clear $2 \mathrm{fi}$ 's, where a resolution $R$ design has no $c$-factor interaction confounded with any other interaction involving less than $R-c$ factors (see [28]), and a $2 \mathrm{fi}$ is called clear if it is not aliased with any main treatment effect and other 2fi's. Cheng and Zhang [29] showed that a $2^{n-m}$ design with $n=N / 4+1$ is a MaxC2 design if and only if it is a GMC design. They also pointed out that, up to isomorphism, the GMC $2^{n-m}$ design with $n=N / 4+1$ can be uniquely expressed as $\mathbf{S}_{N / 4+1}=\left(\mathbf{q}-\mathbf{1}, \mathbf{q}, \mathbf{q}(\mathbf{q}-\mathbf{1}) \mathbf{H}_{q-2}\right)$. It is easy to obtain that

$$
B_{2}\left(\mathbf{S}_{N / 4+1}, \gamma\right)= \begin{cases}1, & \text { for } \boldsymbol{\gamma} \in\left\{(\mathbf{q}-\mathbf{1}) \mathbf{H}_{q-2}, \mathbf{q} \mathbf{H}_{q-2}, \mathbf{q}(\mathbf{q}-\mathbf{1})\right\} \\ \frac{N}{8}-1, & \text { for } \boldsymbol{\gamma} \in \mathbf{H}_{q-2}, \\ 0, & \text { otherwise. }\end{cases}
$$

Therefore,

$$
\begin{aligned}
& { }_{1}^{\#} C_{2}^{(p)}\left(\mathbf{S}_{N / 4+1}\right)= \begin{cases}\frac{N}{4}+1, & \text { for } p=0, \\
0, & \text { otherwise, }\end{cases} \\
& { }_{2}^{\#} C_{2}^{(p)}\left(\mathbf{S}_{N / 4+1}\right)= \begin{cases}2\left(\frac{N}{4}-1\right)+1, & \text { for } p=0, \\
\left(\frac{N}{4}-1\right)\left(\frac{N}{8}-1\right), & \text { for } p=\frac{N}{8}-2, \\
0, & \text { otherwise. }\end{cases}
\end{aligned}
$$

Lemma 1 is a straightforward extension of Lemma A.1, in Appendix, introduced from $[24,25]$.

Lemma 1. Suppose $\mathbf{D}_{b}$ is any s-projection of $\left\{\mathbf{H}_{r}, \boldsymbol{\gamma}, \boldsymbol{\gamma} \mathbf{H}_{r}\right\}$ with $2^{k} \leq s \leq 2^{k+1}-1$ for some $k(0 \leq k \leq r-1)$, where $\gamma$ is independent of the columns of $\mathbf{H}_{r}$. We have

(i) If $\mathbf{D}_{b} \cap\left\{\boldsymbol{\gamma}, \boldsymbol{\gamma} \mathbf{H}_{r}\right\}=\varnothing$, then $\#\left\{U\left(\mathbf{D}_{b}\right) \cap \mathbf{H}_{r}\right\} \geq 2^{k+1}-1$ and the equality holds when $\mathbf{D}_{b}$ has $k+1$ independent columns.

(ii) If $\mathbf{D}_{b} \cap\left\{\boldsymbol{\gamma}, \boldsymbol{\gamma} \mathbf{H}_{r}\right\} \neq \varnothing$, then $\#\left\{U\left(\mathbf{D}_{b}\right) \cap \mathbf{H}_{r}\right\} \geq 2^{k}-1$ and the equality holds when $\mathbf{D}_{b}$ has $k+1$ independent columns.

(iii) If $\mathbf{D}_{b} \cap\left\{\boldsymbol{\gamma}, \boldsymbol{\gamma} \mathbf{H}_{r}\right\} \neq \varnothing$, then $\#\left\{U\left(\mathbf{D}_{b}\right) \cap\left\{\boldsymbol{\gamma}, \boldsymbol{\gamma} \mathbf{H}_{r}\right\}\right\} \geq 2^{k}$ and the equality holds when $\mathbf{D}_{b}$ has $k+1$ independent columns.

(iv) If $\mathbf{D}_{b} \subset \mathbf{H}_{k+1}$, then $U\left(\mathbf{D}_{b}\right)=\mathbf{H}_{k+1}$.

(v) If $\mathbf{D}_{b} \subset\left\{\mathbf{H}_{k}, \boldsymbol{\gamma}, \boldsymbol{\gamma} \mathbf{H}_{k}\right\}$, then $U\left(\mathbf{D}_{b}\right)=\left\{\mathbf{H}_{k}, \boldsymbol{\gamma}, \boldsymbol{\gamma} \mathbf{H}_{k}\right\}$.

Lemma 2 provides a necessary condition for a $2^{n-m}: 2^{s}$ design $\mathbf{D}=\left(\mathbf{D}_{t}: \mathbf{D}_{b}\right)$ with $n=N / 4+1$ to be a $B^{2}$-GMC design.

Lemma 2. Suppose $\mathbf{D}=\left(\mathbf{D}_{t}: \mathbf{D}_{b}\right)$ is a $2^{n-m}: 2^{s}$ design with $n=N / 4+1$ and $2^{k} \leq s \leq 2^{k+1}-1$ for some $k(0 \leq k \leq q-3)$; then, $\mathbf{D}=\left(\mathbf{D}_{t}: \mathbf{D}_{b}\right)$ is a B $B^{2}-G M C$ design only if $\mathbf{D}_{t}=\mathbf{S}_{N / 4+1}$.

Proof. Let $\widetilde{\mathbf{D}}=\left(\mathbf{S}_{\mathrm{N} / 4+1}: \widetilde{\mathbf{D}}_{b}\right)$ be a $2^{n-m}: 2^{s}$ design and $\widetilde{\mathbf{D}}_{b} \in \mathbf{H}_{q-2}$. From (8)-(10), we can obtain

$$
{ }_{1}^{\#} C_{2}(\widetilde{\mathbf{D}})=\left(\frac{N}{4}+1,0, \ldots, 0\right),
$$

which is sequentially maximized by $\widetilde{\mathbf{D}}$, and ${ }_{2}^{\#} C_{2}^{(0)}(\widetilde{\mathbf{D}})=$ $2(N / 4-1)+1=N / 2-1$. Suppose that any $\mathbf{D}=\left(\mathbf{S}_{N / 4+1}\right.$ : $\mathbf{D}_{b}$ ) is not a $B^{2}-\mathrm{GMC} 2^{n-m}: 2^{s}$ design for $n=N / 4+1$. Then, 
by the definition of $B^{2}$-GMC criterion, there should be a $2^{n-m}: 2^{s} \quad$ design $\quad \mathbf{D}^{*}=\left(\mathbf{D}_{t}^{*}: \mathbf{D}_{b}^{*}\right) \quad$ outperforming $\widetilde{D}=\left(\mathbf{S}_{N / 4+1}: \widetilde{D}_{b}\right)$ in terms of (4). This leads to

$$
{ }_{1}^{\#} C_{2}\left(\mathbf{D}^{*}\right)=\left(\frac{N}{4}+1,0, \ldots, 0\right),
$$

and ${ }_{2}^{\#} C_{2}^{(0)}\left(\mathbf{D}^{*}\right) \geq(N / 2)-1$. Clearly,

$$
{ }_{1}^{\#} C_{2}\left(\mathbf{D}_{t}^{*}\right)={ }_{1}^{\#} C_{2}\left(\mathbf{D}^{*}\right)=\left(\frac{N}{4}+1,0, \ldots, 0\right),
$$

and ${ }_{2}^{\#} C_{2}^{(0)}\left(\mathbf{D}_{t}^{*}\right) \geq_{2}^{\#} C_{2}^{(0)}\left(\mathbf{D}^{*}\right) \geq N / 2-1$ noting that $\mathbf{D}_{t}^{*}$ is the unblocked part of $\mathbf{D}^{*}$ and $\mathbf{D}_{t}^{*}$ has $n=N / 4+1$ columns.

In fact, the inequality in the formula ${ }_{2}^{\#} C_{2}^{(0)}\left(\mathbf{D}_{t}^{*}\right) \geq N / 2-$ 1 is not valid. Recall that the MaxC2 design $\mathbf{S}_{N / 4+1}$ has the largest number, $N / 2-1$, of clear 2 fi's among all the $2^{n-m}$ designs with $n=N / 4+1$. Therefore, $\mathbf{D}_{t}^{*}$ has at most $N / 2-1$ clear 2fi's, i.e., ${ }_{2}^{\#} C_{2}^{(0)}\left(\mathbf{D}_{t}^{*}\right)=N / 2-1$. Wu and $\mathrm{Wu}$ [30] showed that a $2^{n-m}$ design with $n=N / 4+1$ is a MaxC2 design if and only if this design has N/2-1 clear 2fi's. This obtains that $\mathbf{D}_{t}^{*}=\mathbf{S}_{N / 4+1}$ up to isomorphism.

With Lemma 2, Theorem 1 provides the constructions of $B^{2}$-GMC designs with $n=N / 4+1$.

Theorem 1. Suppose $\mathbf{D}=\left(\mathbf{D}_{t}: \mathbf{D}_{b}\right)$ is a $2^{n-m}: 2^{s}$ design with $n=N / 4+1$ and $2^{k} \leq s \leq 2^{k+1}-1$ for some $k(0 \leq k \leq q-3)$; then, $\mathbf{D}=\left(\mathbf{D}_{t}: \mathbf{D}_{b}\right)$ is a $B^{2}-G M C$ design if $\mathbf{D}_{t}=\mathbf{S}_{N / 4+1}$ and $\mathbf{D}_{b}$ is any s-projection of $\mathbf{H}_{k+1}$.

Proof. By Lemma 2, if $\mathbf{D}=\left(\mathbf{D}_{t}: \mathbf{D}_{b}\right)$ is a $B^{2}$-GMC $2^{n-m}: 2^{s}$ design with $n=N / 4+1$, then $\mathbf{D}_{t}=\mathbf{S}_{N / 4+1}$ up to isomorphism. Thus, ${ }_{1}^{\#} C_{2}(\mathbf{D})=(N / 4+1,0, \ldots, 0)$ which is sequentially maximized by $\mathbf{D}=\left(\mathbf{S}_{\mathrm{N} / 4+1}: \mathbf{D}_{b}\right)$. Let $u_{1}=$ $\#\left\{U\left(\mathbf{D}_{b}\right) \cap\left\{(\mathbf{q}-\mathbf{1}) \mathbf{H}_{q-2}, \mathbf{q} \mathbf{H}_{q-2}, \mathbf{q}(\mathbf{q}-\mathbf{1})\right\}\right\} \quad$ and $\quad u_{2}=\#$ $\left\{U\left(\mathbf{D}_{b}\right) \cap \mathbf{H}_{q-2}\right\}$; then, from (10), we have

$$
{ }_{2}^{\#} C_{2}^{(p)}(\mathbf{D})= \begin{cases}2\left(\frac{N}{4}-1\right)+1-u_{1}, & \text { for } p=0, \\ \left(\frac{N}{4}-1-u_{2}\right)\left(\frac{N}{8}-1\right), & \text { for } p=\frac{N}{8}-2, \\ 0, & \text { otherwise. }\end{cases}
$$

If $\mathbf{D}$ is a $B^{2}$-GMC design, then $\mathbf{D}$ must sequentially minimize $\left(u_{1}, u_{2}\right)$. There are two different ways to choose $\mathbf{D}_{b}$ from $\mathbf{H}_{q} \backslash \mathbf{S}_{\mathrm{N} / 4+1}$ :

(i) $\mathbf{D}_{b} \cap\left\{(\mathbf{q}-\mathbf{1}) \mathbf{H}_{q-2}, \mathbf{q} \mathbf{H}_{q-2}, \mathbf{q}(\mathbf{q}-\mathbf{1})\right\}=\varnothing$.

(ii) $\mathbf{D}_{b} \cap\left\{(\mathbf{q}-\mathbf{1}) \mathbf{H}_{q-2}, \mathbf{q} \mathbf{H}_{q-2}, \mathbf{q}(\mathbf{q}-\mathbf{1})\right\} \neq \varnothing$.

It is not hard to verify that $\mathbf{D}_{b}$ in case (ii) results in $u_{1}>0$ while $\mathbf{D}_{b}$ in case (i) gives $u_{1}=0$. Therefore, if $\mathbf{D}=\left(\mathbf{S}_{N / 4+1}: \mathbf{D}_{b}\right)$ is a $B^{2}$-GMC design, then $\mathbf{D}_{b}$ must be of case (i). Recall that $\mathbf{D}_{b} \cap \mathbf{S}_{N / 4+1}=\varnothing$, and we have $\mathbf{D}_{b} \subset \mathbf{H}_{q-2}$. By Lemma 1 (i), if $\mathbf{D}_{b} \subset \mathbf{H}_{q-2}$, then $u_{2}=$ \# $\left\{U\left(\mathbf{D}_{b}\right) \cap \mathbf{H}_{q-2}\right\} \geq 2^{k+1}-1$ and the equality holds when
$\mathbf{D}_{b} \subset \mathbf{H}_{k+1}$ up to isomorphism. This completes the proof.

The following example illustrates the construction method in Theorem 1.

Example 1. Consider the construction of the $B^{2}$-GMC $2^{9-4}: 2^{5}$ design. Here, $n=9, m=4, q=5, N=32$, and $s=5$ which leads to $k=2$. According to Theorem 1 , let $\mathbf{D}_{t}=\left(\mathbf{4}, \mathbf{5}, \mathbf{4 5} \mathbf{H}_{3}\right)$ and $\mathbf{D}_{b}$ be any 5-projection of $\mathbf{H}_{3}$, say $\mathbf{D}_{b}=(\mathbf{1}, \mathbf{2}, \mathbf{1 2}, \mathbf{3}, \mathbf{1 3})$. Then, $\mathbf{D}=\left(\mathbf{D}_{t}: \mathbf{D}_{b}\right)$ is a $B^{2}$-GMC $2^{9-4}: 2^{5}$ design.

3.2. $B^{2}$-GMC Designs with $N / 4+1<n \leq 5 N / 16$. To sequentially maximize (4), the first part ${ }_{1}^{\#} C_{2}$ (D) of (4) should be first maximized. Recall that ${ }_{1}^{\#} C_{2}(\mathbf{D})={ }_{1}^{\#} C_{2}\left(\mathbf{D}_{t}\right)$. If $\mathbf{D}_{t}$ has resolution IV, then ${ }_{1}^{\#} C_{2}(\mathrm{D})$ must be maximized. According to [31], when $N / 4+1<n \leq 5 N / 16$, the $\mathbf{D}_{t}$ with resolution IV must be an $n$-projection of some second-order saturated (SOS) designs. In the following, we first review the concept of SOS design.

A $2^{n-m}$ design is called an SOS design if all of its degrees of freedom can be used to estimate only the main treatment effects and 2fi's. In terms of coding theory and projective geometry, Davydov and Tombak [32] showed that, given $N$, only the SOS designs of $N / 4+1, N\left(2^{w-2}+1\right) / 2^{w}$ with $w \geq 4$ and $N / 2$ factors exist. Block and Mee [31] further showed that an SOS design of $N\left(2^{w-2}+1\right) / 2^{w}$ factors can be obtained by doubling some smaller SOS $2^{\left(2^{w-2}+1\right)-\left(2^{w-2}+1-w\right)}$ designs $q-w$ times. Zhang and Cheng [33] showed that the SOS design of $N / 2$ factors can be uniquely represented by $\mathbf{S}_{N / 2}=\left(\mathbf{q}, \mathbf{q H}_{q-1}\right)$ up to isomorphism. Let

$\mathscr{L}(w)=\left\{D^{q-w}(\mathbf{Y}): \mathbf{Y}\right.$ is an $\operatorname{SOS} 2^{\left(2^{w-2}+1\right)-\left(2^{w-2}+1-w\right)}$ design $\}$,

denote the collection of all the SOS designs obtained by doubling some SOS $2^{\left(2^{w-2}+1\right)-\left(2^{w-2}+1-w\right)}$ designs $q-w$ times. Especially, in $\mathscr{L}(w)$, we denote the SOS design obtained by doubling the MaxC2 $2^{\left(2^{w-2}+1\right)-\left(2^{w-2}+1-w\right)}$ design $\Phi(w)=\left((\mathbf{w}-\mathbf{1})_{2^{w}}, \mathbf{w}_{2^{w}}, \mathbf{w}_{2^{w}}(\mathbf{w}-\mathbf{1})_{2^{w}} \mathbf{H}_{w-2}^{w}\right)$ as $\mathbf{S}_{N\left(2^{w-2}+1\right) / 2^{w}}$.

With a little algebra, it is easy to verify that

$$
\begin{aligned}
D^{q-w}\left(\mathbf{I}_{2^{w}}\right) & =\left(\mathbf{I}_{2^{q}}, \mathbf{H}_{q^{-w}}^{q}\right)=\left(\mathbf{I}, \mathbf{H}_{q-w}\right) \\
D^{q-w}\left(\mathbf{1}_{2^{w}}\right) & =(\mathbf{q}-\mathbf{w}+\mathbf{1})_{2^{q}}\left(\mathbf{I}_{2^{q}}, \mathbf{H}_{q-w}^{q}\right) \\
& =(\mathbf{q}-\mathbf{w}+\mathbf{1})\left(\mathbf{I}, \mathbf{H}_{q-w}\right) \\
D^{q-w}\left(\mathbf{2}_{2^{w}}\right) & =(\mathbf{q}-\mathbf{w}+\mathbf{2})_{2^{q}}\left(\mathbf{I}_{2^{q}}, \mathbf{H}_{q-w}^{q}\right) \\
& =(\mathbf{q}-\mathbf{w}+\mathbf{2})\left(\mathbf{I}, \mathbf{H}_{q-w}\right) \\
& \vdots \\
D^{q-w}\left((\mathbf{w}-\mathbf{1})_{2^{w}}\right) & =(\mathbf{q}-\mathbf{1})_{2^{q}}\left(\mathbf{I}_{2^{q}}, \mathbf{H}_{q-w}^{q}\right)=(\mathbf{q}-\mathbf{1})\left(\mathbf{I}, \mathbf{H}_{q-w}\right) \\
D^{q-w}\left(\mathbf{w}_{2^{w}}\right) & =\mathbf{q}_{2^{q}}\left(\mathbf{I}_{2 q}, \mathbf{H}_{q-w}^{q}\right)=\mathbf{q}\left(\mathbf{I}, \mathbf{H}_{q-w}\right),
\end{aligned}
$$

where $\mathbf{I}_{2^{q-w}} \otimes \mathbf{1}_{2^{w}}=(\mathbf{q}-\mathbf{w}+\mathbf{1})_{2^{q}}, \mathbf{I}_{2^{q-w}} \otimes \mathbf{2}_{2^{w}}=(\mathbf{q}-\mathbf{w}+\mathbf{2})_{2^{q}}$, $\ldots, \mathbf{I}_{2^{q-w}} \otimes(\mathbf{w}-\mathbf{1})_{2^{w}}=(\mathbf{q}-\mathbf{1})_{2^{q}}, \quad$ and $\quad \mathbf{I}_{2^{q-w}} \otimes \mathbf{w}_{2^{w}}=\mathbf{q}_{2^{q}}$. Then, 


$$
D^{q-w}\left((\mathbf{w}-\mathbf{1})_{2^{w}} \mathbf{w}_{2^{w}} \mathbf{H}_{w-2}^{w}\right)=\mathbf{q}(\mathbf{q}-\mathbf{1})\left(\mathbf{H}_{q-2} \backslash \mathbf{H}_{q-w}\right) .
$$

Therefore, $\mathbf{S}_{N\left(2^{w-2}+1\right) / 2^{w}}$ can be expressed as

$$
\begin{aligned}
\mathbf{S}_{N\left(2^{w-2}+1\right) / 2^{w}}= & D^{q-w}(\Phi(w)) \\
= & \left((\mathbf{q}-\mathbf{1}),(\mathbf{q}-\mathbf{1}) \mathbf{H}_{q-w}, \mathbf{q}, \mathbf{q} \mathbf{H}_{q-w}, \mathbf{q}(\mathbf{q}-\mathbf{1})\right. \\
& \left.\left(\mathbf{H}_{q-2} \backslash \mathbf{H}_{q-w}\right)\right) .
\end{aligned}
$$

The following lemma provides a necessary condition for a $2^{n-m}: 2^{s}$ design $\mathbf{D}=\left(\mathbf{D}_{t}: \mathbf{D}_{b}\right)$ with $N / 4+1<n \leq 5 N / 16$ to be a $B^{2}$-GMC design.

Lemma 3. Suppose $\mathbf{D}=\left(\mathbf{D}_{t}: \mathbf{D}_{b}\right)$ is a $2^{n-m}: 2^{\text {s }}$ design with $N\left(2^{w-1}+1\right) / 2^{w+1}<n \leq N\left(2^{w-2}+1\right) / 2^{w}$ for $w \geq 4$ and $2^{k} \leq s \leq 2^{k+1}-1 \quad$ for some $k(0 \leq k \leq q-3)$; then, $\mathbf{D}=\left(\mathbf{D}_{t}: \mathbf{D}_{b}\right)$ is a $B^{2}-G M C$ design only if $\mathbf{D}_{t} \subset \mathbf{S}_{N\left(2^{w-2}+1\right) / 2^{w}}$.

Proof. As discussed in the first paragraph of this section, if $\mathbf{D}=\left(\mathbf{D}_{t}: \mathbf{D}_{b}\right)$ is a $B^{2}$-GMC $2^{n-m}: 2^{s}$ design with $N\left(2^{w-1}+1\right) / 2^{w+1}<n \leq N\left(2^{w-2}+1\right) / 2^{w}$, then $\mathbf{D}_{t}$ must be an $n$-projection of some SOS designs in $\mathscr{L}(v)$ with $4 \leq v \leq w$ or $\mathbf{S}_{\mathrm{N} / 2}$.

Let $\mathbf{P}_{1}$ be an $n$-projection of $\mathbf{S}_{N / 2}$. According to Theorem 3 in [33], we obtain

$$
{ }_{2}^{\#} C_{2}^{(p)}\left(\mathbf{P}_{1}\right)= \begin{cases}0, & \text { for } p<n-\frac{N}{4}-1, \\ \frac{N}{4}\left(n-\frac{N}{4}\right), & \text { for } p=n-\frac{N}{4}-1, \\ 0, & \text { for } n-\frac{N}{4}-1<p<\frac{N}{8}-1 .\end{cases}
$$

Let $\quad \mathbf{X}_{v}=\left(\mathbf{a}_{1}, \mathbf{a}_{2}, \ldots, \mathbf{a}_{2^{v-2}+1}\right)$ be any SOS $2^{\left(2^{v-2}+1\right)-\left(2^{v-2}+1-v\right)}$ design but not a MaxC2 design; then, $D^{q-v}\left(\mathbf{X}_{v}\right) \in \mathscr{L}(v)$. Denote $p_{i}$ as the number of clear 2fi's of $\mathbf{X}_{v}$ involving $\mathbf{a}_{i}$ for $i=1,2, \ldots, 2^{v-2}+1$ and $c$ as the total number of clear 2fi's of $\mathbf{X}_{v}$. Let $\mathbf{P}_{v}$ be an $n$-projection of $D^{q^{-v}}\left(\mathbf{X}_{v}\right) \quad$ and $\quad \overline{\mathbf{P}}_{v}=D^{q^{-v}}\left(\mathbf{X}_{v}\right) \backslash \mathbf{P}_{v}$. Then, $\# \overline{\mathbf{P}}_{v}=N$ $\left(2^{v-2}+1\right) / 2^{v}-n \leq N / 2^{v}$. Applying equations (23) and (24) in the proof of Theorem 3.1 of [29], among all the $n$-projections of $D^{q^{-v}}\left(\mathbf{X}_{v}\right), \mathbf{P}_{v}$ sequentially maximizes ${ }_{2}^{\#} C_{2}$ in (5), only if $\overline{\mathbf{P}}_{v} \subset D^{q^{-v}}\left(\mathbf{a}_{j}\right)$, where $\mathbf{a}_{j}$ is the column such that $p_{j}=\max \left\{p_{1}, p_{2}, \ldots, p_{2^{v-2}+1}\right\}$. For a $\mathbf{P}_{v}$ with $\overline{\mathbf{P}}_{v} \subset D^{q-v}\left(\mathbf{a}_{j}\right)$, according to equation (24) in the proof of Theorem 3.1 of [29], it is obtained that

$$
{ }_{2}^{\#} C_{2}^{(p)}\left(\mathbf{P}_{v}\right)= \begin{cases}0, & \text { for } 0 \leq p<n-\frac{N}{4}-1, \\ \frac{p_{j} N}{2^{v}}(n-N / 4), & \text { for } p=n-\frac{N}{4}-1, \\ 0, & \text { for } n-\frac{N}{4}-1<p<\frac{N}{2^{v}}-1, \\ \left(c-p_{j}\right)\left(\frac{N}{2^{v}}\right)^{2}, & \text { for } p=\frac{N}{2^{v}}-1 .\end{cases}
$$

For a MaxC22 $2^{\left(2^{v-2}+1\right)-\left(2^{v-2}+1-v\right)}$ design, it has $2^{v-1}-1$ clear 2fi's and $p_{j}=2^{v-2}$. Since $\mathbf{X}_{v}$ is not a MaxC2 design, we have

$$
\begin{gathered}
p_{j}=2^{v-2}, \\
c-p_{j}<2^{v-1}-2^{v-2}-1,
\end{gathered}
$$

or

$$
\begin{aligned}
p_{j} & <2^{v-2}, \\
c & <2^{v-1}-1 .
\end{aligned}
$$

Let $\mathbf{Q}_{v}$ be an $n$-projection of $\mathbf{S}_{N\left(2^{v-2}+1\right) / 2^{v}}$ obtained by doubling $\Phi(v)$, the MaxC22 $2^{\left(2^{v-2}+1\right)-\left(2^{v-2}+1-v\right)}$ design as mentioned above. With a column permutation, rewrite $D^{q}(\cdot)$ as

$$
\begin{aligned}
D_{\mathrm{RC}}^{q}(\cdot)= & \left(\mathbf{H}_{q-v}, \mathbf{H}_{q-2} \backslash \mathbf{H}_{q-v},(\mathbf{q}-\mathbf{1})\left(\mathbf{H}_{q-2} \backslash \mathbf{H}_{q^{-v}}\right),\right. \\
& \left.\mathbf{q}\left(\mathbf{H}_{q^{-2}} \backslash \mathbf{H}_{q-v}\right), \mathbf{q}(\mathbf{q}-\mathbf{1})\left(\mathbf{I}, \mathbf{H}_{q-v}\right), \mathbf{S}_{N\left(2^{v-2}+1\right) / 2^{v}}\right),
\end{aligned}
$$

in a re-changed Yates order. Write $\Phi(v)$ as $\Phi(v)=\left(\mathbf{s}_{1}, \mathbf{s}_{2}, \ldots, \mathbf{s}_{2^{v-2}+1}\right)$, where $\mathbf{s}_{1}=(\mathbf{v}-\mathbf{1})_{2^{v}}, \mathbf{s}_{2}=\mathbf{v}_{2^{v}}$ and $\left(\mathbf{s}_{3}, \ldots, \mathbf{s}_{2^{v-2}+1}\right)=\mathbf{v}_{2^{v}}(\mathbf{v}-\mathbf{1})_{2^{v}} \mathbf{H}_{v-2}^{v}$. Applying equations (23) and (24) in the proof of Theorem 3.1 of [29], among all the $n$-projections of $\mathbf{S}_{N\left(2^{v-2}+1\right) / 2^{v}}, \mathbf{Q}_{v}$ sequentially maximizes ${ }_{2}^{\#} C_{2}$ in (3), only if $\overline{\mathbf{Q}}_{v} \subset D^{q-v}\left(\mathbf{s}_{i}\right)$ with $i=1$ or 2 . In the following, we investigate ${ }_{2}^{\#} C_{2}^{(p)}\left(\mathbf{Q}_{v}\right)$ with $\overline{\mathbf{Q}}_{v} \subset D^{q^{-v}}\left(\mathbf{s}_{1}\right)$ for which the following analysis and final conclusion are the same as that for ${ }_{2}^{\#} C_{2}^{(p)}\left(\mathbf{Q}_{v}\right)$ with $\overline{\mathbf{Q}}_{v} \subset D^{q^{-v}}\left(\mathbf{s}_{2}\right)$. For $\mathbf{Q}_{v}$ with $\overline{\mathbf{Q}}_{v} \subset D^{q^{-v}}\left(\mathbf{s}_{1}\right)$, from equations (18) and (23), it is obtained that 


$$
B_{2}\left(\mathbf{Q}_{v}, \boldsymbol{\gamma}\right)= \begin{cases}0, & \text { for } \boldsymbol{\gamma} \in \mathbf{S}_{N\left(2^{v-2}+1\right) / 2^{v}}, \\ n-\frac{N}{4}, & \text { for } \boldsymbol{\gamma} \in\left\{\mathbf{q}\left(\mathbf{H}_{q-2} \backslash \mathbf{H}_{q-v}\right), \mathbf{q}(\mathbf{q}-\mathbf{1})\left(\mathbf{I}, \mathbf{H}_{q-v}\right)\right\} \\ \frac{N}{2^{v}}, & \text { for } \boldsymbol{N} \in(\mathbf{q}-\mathbf{1})\left(\mathbf{H}_{q-2} \backslash \mathbf{H}_{q-v}\right), \\ \frac{N}{2^{v}}, & \text { for } \boldsymbol{N} \in \mathbf{H}_{q-2} \backslash \mathbf{H}_{q-v}, \\ B_{2}\left(\bar{Q}_{v}, \boldsymbol{\gamma}\right)+\frac{N}{8}, & \text { for } \boldsymbol{\gamma} \in \mathbf{H}_{q-v},\end{cases}
$$

where the second equality can be easily verified by noting that

$$
\begin{aligned}
& \left\{\mathbf{q}\left(\mathbf{H}_{q-2} \backslash \mathbf{H}_{q-v}\right), \mathbf{q}(\mathbf{q}-\mathbf{1})\left(\mathbf{I}, \mathbf{H}_{q-v}\right)\right\} \\
= & \left\{D^{q-v}\left(\mathbf{s}_{1} \mathbf{s}_{2}\right), D^{q-v}\left(\mathbf{s}_{1} \mathbf{s}_{3}\right), \ldots, D^{q-v}\left(\mathbf{s}_{1} \mathbf{s}_{2^{v-2}+1}\right)\right\} .
\end{aligned}
$$

$$
{ }_{2}^{\#} C_{2}^{(p)}\left(\mathbf{Q}_{v}\right)= \begin{cases}0, & \text { for } 0 \leq p<n-\frac{N}{4}-1, \\ \frac{N}{4}(n-N / 4), & \text { for } p=n-\frac{N}{4}-1, \\ 0, & \text { for } n-\frac{N}{4}-1<p<\frac{N}{2^{v}}-1 \\ \frac{N}{2^{v}}\left(N / 4-N / 2^{v}\right), & \text { for } p=\frac{N}{2^{v}}-1, \\ 0, & \text { for } \frac{N}{2^{v}}-1<p<\frac{N}{8}-\frac{N}{2^{v}}-1 \\ \left(\frac{N}{4}-\frac{N}{2^{v}}\right)\left(\frac{N}{8}-\frac{N}{2^{v}}\right), & \text { for } p=\frac{N}{8}-\frac{N}{2^{v}}-1 .\end{cases}
$$

Denote $\mathbf{D}^{*}=\left(\mathbf{D}_{t}^{*}: \mathbf{D}_{b}^{*}\right)$, where $\mathbf{D}_{t}^{*} \subset \mathbf{S}_{N\left(2^{w-2}+1\right) / 2^{w}}$, $\mathbf{S}_{N\left(2^{w-2}+1\right) / 2^{w}} \backslash \mathbf{D}_{t}^{*} \subset D^{q-w}\left(\mathbf{s}_{1}\right)$ and $\mathbf{D}_{b}^{*} \subset \mathbf{H}_{k+1}$. Since $k \leq q-3$,
Therefore,

(n)


Comparing (27) with (19), (20) for $4 \leq v \leq w$, and (26) for $4 \leq v \leq w-1$, it is obtained that if $\mathbf{D}=\left(\mathbf{D}_{t}: \mathbf{D}_{b}\right)$ is a $B^{2}-\mathrm{GMC}$ design, $\mathbf{D}$ should not be worse than $\mathbf{D}^{*}$ in terms of (3). Therefore, if $\mathbf{D}=\left(\mathbf{D}_{t}: \mathbf{D}_{b}\right)$ is a $B^{2}$-GMC design, $\mathbf{D}_{t}$ should be an $n$-projection of $\mathbf{S}_{N\left(2^{w-2}+1\right) / 2^{w}}$. This completes the proof.

The lemma below is an extension of Lemma A.2, in Appendix, introduced from [34].

Lemma 4. Suppose $\mathbf{D}_{t}$ consists of the last $n$, $N\left(2^{w-1}+1\right) / 2^{w+1}<n \leq N\left(2^{w-2}+1\right) / 2^{w}, \quad$ columns of $\mathbf{S}_{N\left(2^{w-2}+1\right) / 2^{w}}$; then, $B_{2}\left(\mathbf{D}_{t}, \gamma_{1}\right) \geq B_{2}\left(\mathbf{D}_{t}, \gamma_{2}\right)$ if $\gamma_{1}$ is ahead of $\gamma_{2}$ in $\mathbf{H}_{q-w}$.

Proof. Suppose $\mathbf{O}$ consists of the last $n-N / 4$ columns of $D^{q-w}\left((\mathbf{w}-\mathbf{1})_{2^{w}}\right)$. Then, $\quad \mathbf{D}_{t}=\left\{\mathbf{O}, \mathbf{S}_{N\left(2^{w-2}+1\right) / 2^{w}} \backslash D^{q-w}\right.$ $(\mathbf{w}-\mathbf{1})\}$. From (16) and (18), for any $\boldsymbol{\gamma} \in \mathbf{H}_{q-w}$, $B_{2}\left(\mathbf{D}_{t}, \boldsymbol{\gamma}\right)=B_{2}(\mathbf{O}, \boldsymbol{\gamma})+N / 8$. Straightforwardly, from Lemma A.2, it is obtained that $B_{2}\left(\mathbf{O}, \gamma_{1}\right) \geq B_{2}\left(\mathbf{O}, \gamma_{2}\right)$ if $\gamma_{1}$ is ahead of $\gamma_{2}$ in $\mathbf{H}_{q-w}$. This completes the proof.

$$
B_{2}\left(\mathbf{D}_{t}^{*}, \gamma\right)=\left\{\begin{array}{l}
0, \\
n-\frac{N}{4}, \\
\frac{N}{2^{w}}, \\
\frac{N}{8}-\frac{N}{2^{w}}, \\
B_{2}\left(\bar{D}_{t}^{*}, \gamma\right)+\frac{N}{8}, \text { for } \gamma \in \mathbf{H}_{q-w} .
\end{array}\right.
$$

Let

$u_{1}=\#\left\{U\left(\mathbf{D}_{b}^{*}\right) \cap\left\{\mathbf{q}\left(\mathbf{H}_{q-2} \backslash \mathbf{H}_{q-w}\right), \mathbf{q}(\mathbf{q}-\mathbf{1})\left(\mathbf{I}, \mathbf{H}_{q-w}\right)\right\}\right\}$,

$u_{2}=\#\left\{U\left(\mathbf{D}_{b}^{*}\right) \cap(\mathbf{q}-\mathbf{1})\left(\mathbf{H}_{q-2} \backslash \mathbf{H}_{q-w}\right)\right\}$,

$u_{3}=\#\left\{U\left(\mathbf{D}_{b}^{*}\right) \cap\left(\mathbf{H}_{q-2} \backslash \mathbf{H}_{q-w}\right)\right\}$,
With Lemmas 3 and 4, Theorem 2 provides the construction methods of $B^{2}$-GMC $2^{n-m}: 2^{s}$ designs with $N / 4+1<n \leq 5 N / 16$.

Theorem 2. Let $\mathbf{D}=\left(\mathbf{D}_{t}: \mathbf{D}_{b}\right)$ be a $2^{n-m}: 2^{s}$ design with $N\left(2^{w-1}+1\right) / 2^{w+1}<n \leq N\left(2^{w-2}+1\right) / 2^{w}$ for $w \geq 4$ and $2^{k} \leq s \leq 2^{k+1}-1$ for some $0 \leq k \leq q-3$. Suppose $2^{r} \leq N$ $\left(2^{w-2}+1\right) / 2^{w}-n \leq 2^{r+1}-1$ for some $0 \leq r \leq q-w-1$; then, $\mathbf{D}$ is a $B^{2}-G M C$ design if $\mathbf{D}_{t}$ consists of the last $n$ columns of $\mathbf{S}_{N\left(2^{w-2}+1\right) / 2^{w}}$ and

(a) $\mathbf{D}_{b}$ is any s-projection of $\left\{\mathbf{H}_{k}, \mathbf{q}-\mathbf{1},(\mathbf{q}-\mathbf{1}) \mathbf{H}_{k}\right\}$ when $k \leq r \leq q-w-1$.

(b) $\mathbf{D}_{b}$ is any s-projection of $\mathbf{H}_{k+1}$ when $r+1 \leq k \leq q-w-1$.

(c) $\mathbf{D}_{b}$ is any s-projection of $\mathbf{H}_{q-2}$ when $q-w-1<k \leq q-3$.

Proof. By Lemma 3 and its proof, if $\mathbf{D}^{*}=\left(\mathbf{D}_{t}^{*}: \mathbf{D}_{b}^{*}\right)$ is a $B^{2}$-GMC $2^{n-m}: 2^{s}$ design, there should be $\mathbf{D}_{t}^{*} \subset \mathbf{S}_{N\left(2^{w-2}+1\right) 2^{w}}$ and $\overline{\mathbf{D}}_{t}^{*}=\mathbf{S}_{N\left(2^{w-2}+1\right) 2^{w}} \backslash \mathbf{D}_{t}^{*} \subset D^{q-w}\left((\mathbf{w}-\mathbf{1})_{2^{w}}\right)$. Substituting $v$ with $w$ in (24), we obtain

$$
\begin{aligned}
& \text { for } \boldsymbol{\gamma} \in \mathbf{S}_{\left(N\left(2^{w-2}+1\right) / 2^{w}\right)}, \\
& \text { for } \boldsymbol{\gamma} \in\left\{\mathbf{q}\left(\mathbf{H}_{q-2} \backslash \mathbf{H}_{q-w}\right), \mathbf{q}(\mathbf{q}-\mathbf{1})\left(\mathbf{I}, \mathbf{H}_{q-w}\right)\right\} \text {, } \\
& \text { for } \boldsymbol{\gamma} \in(\mathbf{q}-\mathbf{1})\left(\mathbf{H}_{q-2} \backslash \mathbf{H}_{q-w}\right) \text {, } \\
& \text { for } \boldsymbol{\gamma} \in \mathbf{H}_{q-2} \backslash \mathbf{H}_{q-w} \text {, }
\end{aligned}
$$$$
\text { and then }
$$ 


$$
{ }_{2}^{\#} C_{2}^{(p)}\left(\mathbf{D}^{*}\right)= \begin{cases}\left(n-\frac{N}{4}\right)\left(\frac{N}{4}-u_{1}\right), & \text { for } p=n-\frac{N}{4}-1, \\ \frac{N}{2^{w}}\left(\frac{N}{4}-\frac{N}{2^{w}}-u_{2}\right), & \text { for } p=\frac{N}{2^{w}}-1, \\ \left(\frac{N}{8}-\frac{N}{2^{w}}\right)\left(\frac{N}{4}-\frac{N}{2^{w}}-u_{3}\right), & \text { for } p=\frac{N}{8}-\frac{N}{2^{w}}-1, \\ f\left(\mathbf{D}_{t}^{*}, p\right), & \text { for } p \geq \frac{N}{8}-1, \\ 0, & \text { otherwise, }\end{cases}
$$

where $\quad f\left(\mathbf{D}_{t}^{*}, p\right)={ }_{2}^{\#} C_{2}^{(p)}\left(\mathbf{D}_{t}^{*}\right)-(p+1) \#\left\{\gamma: \gamma \in \mathbf{H}_{q-}\right.$ $\left.w \cap U\left(\mathbf{D}_{b}^{*}\right), B_{2}\left(\mathbf{D}_{t}^{*}, \gamma\right)=p+1\right\}$. Therefore, $\mathbf{D}^{*}$ sequentially maximizes ${ }_{2}^{\#} C_{2}$ among all the possible $2^{n-m}: 2^{s}$ designs, only if $\mathbf{D}^{*}$ sequentially minimizes

$$
\left(u_{1}, u_{2}, u_{3}\right) \text {. }
$$

For ease of presenting, let $\mathbf{A}=\left\{\mathbf{q}\left(\mathbf{H}_{q-2} \backslash \mathbf{H}_{q-w}\right)\right.$, $\left.\mathbf{q}(\mathbf{q}-\mathbf{1})\left(\mathbf{I}, \mathbf{H}_{q-w}\right)\right\}, \quad \mathbf{B}=(\mathbf{q}-\mathbf{1})\left(\mathbf{H}_{q-2} \backslash \mathbf{H}_{q-w}\right), \quad$ and $\mathbf{C}=$ $\mathbf{H}_{q-2} \backslash \mathbf{H}_{q-w}$.

For (a), when $k \leq q-w-1$, there are two different ways to choose $\mathbf{D}_{b}^{*}$ from $\mathbf{H}_{q}$ : (i) $\mathbf{D}_{b}^{*} \cap\{\mathbf{A}, \mathbf{B}, \mathbf{C}\}=\varnothing$ and (ii) $\mathbf{D}_{b}^{*} \cap\{\mathbf{A}, \mathbf{B}, \mathbf{C}\} \neq \varnothing$. Recall that if $\mathbf{D}^{*}=\left(\mathbf{D}_{t}^{*}: \mathbf{D}_{b}^{*}\right)$ is a $B^{2}$-GMC design, then $\mathbf{D}_{t}^{*} \subset \mathbf{S}_{N\left(2^{w-2}+1\right) 2^{w}}$ with $\bar{D}_{t}^{*}=$ $\mathbf{S}_{N\left(2^{w-2}+1\right) 2^{w}} \backslash \mathbf{D}_{t}^{*} \subset D^{q-w}\left((\mathbf{w}-\mathbf{1})_{2^{w}}\right)$. Case (i) implies that $\mathbf{D}_{b}^{*} \subset\left\{\mathbf{H}_{q-w}, \bar{D}_{t}^{*}\right\}$, and thus $\mathbf{D}_{b}^{*} \subset\left\{\mathbf{H}_{q-w}, \mathbf{q}-\mathbf{1},(\mathbf{q}-\mathbf{1})\right.$ $\left.\mathbf{H}_{q-w}\right\}$. Therefore, choosing $\mathbf{D}_{b}^{*}$ according to case (i) results in $u_{1}=u_{2}=u_{3}=0$. If $\mathbf{D}_{b}^{*}$ is chosen according to case (ii), then $U\left(\mathbf{D}_{b}^{*}\right) \cap\{\mathbf{A}, \mathbf{B}, \mathbf{C}\} \neq \varnothing$ which results in $u_{1}>0, u_{2}>0$, or $u_{3}>0$. Clearly, if $\mathbf{D}^{*}$ is a $B^{2}$-GMC design, $\mathbf{D}_{b}^{*}$ should be chosen as (i). In the following, we consider only $\mathbf{D}_{b}^{*} \cap\{\mathbf{A}, \mathbf{B}, \mathbf{C}\}=\varnothing$, i.e., $\mathbf{D}_{b}^{*} \subset\left\{\mathbf{H}_{q-w},(\mathbf{q}-\mathbf{1}),(\mathbf{q}-\mathbf{1}) \mathbf{H}_{q-w}\right\}$.

For $\mathbf{D}=\left(\mathbf{D}_{t}: \mathbf{D}_{b}\right)$ in $(\mathrm{a})$, according to $(\mathrm{v})$ in Lemma 1 , if $\mathbf{D}_{b} \subset\left\{\mathbf{H}_{k},(\mathbf{q}-\mathbf{1}),(\mathbf{q}-\mathbf{1}) \mathbf{H}_{k}\right\}$ with $k \leq q-w-1$, then $U\left(\mathbf{D}_{b}\right)=\left\{\mathbf{H}_{k},(\mathbf{q}-\mathbf{1}),(\mathbf{q}-\mathbf{1}) \mathbf{H}_{k}\right\}$. Denote $\mathbf{b}$ as the last column of $\mathbf{H}_{k}$ in Yates order, and $B_{2}\left(\mathbf{D}_{t}, \mathbf{b}\right)=b$; then, $b \geq N / 8$. According to Lemma 4 , for $\boldsymbol{\gamma} \in \mathbf{H}_{q-w}$, if $\boldsymbol{\gamma}$ is ahead of $\mathbf{b}$ in $\mathbf{H}_{q-w}$, then $B_{2}\left(\mathbf{D}_{t}, \gamma\right) \geq b$ by (18). For any $\boldsymbol{\gamma} \in\left\{(\mathbf{q}-\mathbf{1}),(\mathbf{q}-\mathbf{1}) \mathbf{H}_{k}\right\}$, we have $B_{2}\left(\mathbf{D}_{t}, \boldsymbol{\gamma}\right)=0$. For any $\boldsymbol{\gamma}$ such that $B_{2}\left(\mathbf{D}_{t}, \gamma\right)<b$, we have $\gamma \in \mathbf{H}_{q} \backslash \mathbf{H}_{k}$. Therefore, for $p \leq b-2$,

$$
\begin{aligned}
{ }_{2}^{\#} C_{2}^{(p)}(\mathbf{D}) & =(p+1) \#\left\{\gamma \in \mathbf{H}_{q}: \gamma \notin U\left(\mathbf{D}_{b}\right), B_{2}\left(\mathbf{D}_{t}, \gamma\right)=p+1\right\} \\
& =(p+1) \#\left\{\gamma \in \mathbf{H}_{q}: B_{2}\left(\mathbf{D}_{t}, \boldsymbol{\gamma}\right)=p+1\right\} \\
& ={ }_{2}^{\#} C_{2}^{(p)}\left(\mathbf{D}_{t}\right),
\end{aligned}
$$

and for $p \geq b$,

$$
\begin{aligned}
{ }_{2}^{\#} C_{2}^{(p)}(\mathbf{D}) & =(p+1) \#\left\{\gamma \in \mathbf{H}_{q}: \gamma \notin U\left(\mathbf{D}_{b}\right), B_{2}\left(\mathbf{D}_{t}, \gamma\right)=p+1\right\} \\
& =(p+1) \#\left\{\gamma \in \mathbf{H}_{q}: B_{2}\left(\mathbf{D}_{t}, \gamma\right)=p+1\right\}-(p+1) \#\left\{\gamma \in \mathbf{H}_{k}: B_{2}\left(\mathbf{D}_{t}, \gamma\right)=p+1\right\} \\
& =0
\end{aligned}
$$

From (33), it is obtained that

$$
\begin{aligned}
& \left({ }_{2}^{\#} C_{2}^{(0)}(\mathbf{D}), \ldots,{ }_{2}^{\#} C_{2}^{(N / 8-1)}(\mathbf{D}),{ }_{2}^{\#} C_{2}^{(N / 8)}(\mathbf{D}), \ldots,{ }_{2}^{\#} C_{2}^{(b-2)}(\mathbf{D})\right) \\
= & \left({ }_{2}^{\#} C_{2}^{(0)}\left(\mathbf{D}_{t}\right), \ldots,{ }_{2}^{\#} C_{2}^{(N / 8-1)}\left(\mathbf{D}_{t}\right),{ }_{2}^{\#} C_{2}^{(N / 8)}\left(\mathbf{D}_{t}\right), \ldots,{ }_{2}^{\#} C_{2}^{(b-2)}\left(\mathbf{D}_{t}\right)\right) .
\end{aligned}
$$

Note that $\mathbf{D}_{t}$ is a GMC design [29]; then, $\mathbf{D}$ maximizes

$$
\left({ }_{2}^{\#} C_{2}^{(0)}(\cdot), \ldots,{ }_{2}^{\#} C_{2}^{(N / 8-1)}(\cdot),{ }_{2}^{\#} C_{2}^{(N / 8)}(\cdot), \ldots,{ }_{2}^{\#} C_{2}^{(b-2)}(\cdot)\right),
$$

among all the possible $2^{n-m}: 2^{s}$ designs.

Suppose $\mathbf{D}$ is not a $B^{2}$-GMC design; then, there should be a $\mathbf{D}^{*}=\left(\mathbf{D}_{t}^{*}: \mathbf{D}_{b}^{*}\right)$ which outperforms $\mathbf{D}=\left(\mathbf{D}_{t}: \mathbf{D}_{b}\right)$ in terms of (3). This implies that there exists some $p_{1} \geq b-1$ such that 


$$
\begin{aligned}
&\left({ }_{2}^{\#} C_{2}^{(0)}\left(\mathbf{D}^{*}\right), \ldots,{ }_{2}^{\#} C_{2}^{(N / 8-1)}\left(\mathbf{D}^{*}\right),{ }_{2}^{\#} C_{2}^{(N / 8)}\left(\mathbf{D}^{*}\right), \ldots,{ }_{2}^{\#} C_{2}^{(b-2)}\left(\mathbf{D}^{*}\right)\right) \\
&=\left({ }_{2}^{\#} C_{2}^{(0)}(\mathbf{D}), \ldots,{ }_{2}^{\#} C_{2}^{(N / 8-1)}(\mathbf{D}),{ }_{2}^{\#} C_{2}^{(N / 8)}(\mathbf{D}), \ldots,{ }_{2}^{\#} C_{2}^{(b-2)}(\mathbf{D})\right), \\
&{ }_{2}^{\#} C_{2}^{\left(p_{1}-1\right)}\left(\mathbf{D}^{*}\right)>{ }_{2}^{\#} C_{2}^{\left(p_{1}-1\right)}(\mathbf{D}) .
\end{aligned}
$$
have

Recalling the definitions of ${ }_{2}^{\#} C_{2}^{(p)}(\mathbf{D})$ and $B_{2}\left(\mathbf{D}_{t}, \gamma\right)$, we

$$
\begin{aligned}
\sum_{p=0}^{P} \frac{{ }_{2}^{\#} C_{2}^{(p)}(\mathbf{D})}{(p+1)}= & \sum_{p=0}^{P} \#\left\{\gamma \in \mathbf{H}_{q}: \gamma \notin U\left(\mathbf{D}_{b}\right), B_{2}\left(\mathbf{D}_{t}, \boldsymbol{\gamma}\right)=p+1\right\} \\
= & \sum_{p=0}^{P} \#\left\{\gamma \in\left(\mathbf{H}_{q} \backslash \mathbf{S}_{N\left(2^{w-2}+1\right) / 2^{w}}\right): B_{2}\left(\mathbf{D}_{t}, \gamma\right)=p+1\right\} \\
& -\sum_{p=0}^{P} \#\left\{\gamma \in U\left(\mathbf{D}_{b}\right): B_{2}\left(\mathbf{D}_{t}, \boldsymbol{\gamma}\right)=p+1\right\} \\
= & \#\left(\mathbf{H}_{q} \backslash \mathbf{S}_{N\left(2^{w-2}+1\right) / 2^{w}}\right)-\# \mathbf{H}_{k} \\
= & 2^{q}-\frac{N\left(2^{w-2}+1\right)}{2^{w}}-2^{k},
\end{aligned}
$$

where the second and third equalities are due to $B_{2}\left(\mathbf{D}_{t}, \boldsymbol{\gamma}\right)=$ 0 for any $\boldsymbol{\gamma} \in \mathbf{S}_{N\left(2^{w-2}+1\right) / 2^{w}}$. Similarly,

$$
\begin{aligned}
& \sum_{p=0}^{P} \frac{{ }_{2}^{\#} C_{2}^{(p)}\left(\mathbf{D}^{*}\right)}{(p+1)}=\sum_{p=0}^{P} \#\left\{\boldsymbol{\gamma} \in \mathbf{H}_{q}: \boldsymbol{\gamma} \notin U\left(\mathbf{D}_{b}^{*}\right), B_{2}\left(\mathbf{D}_{t}^{*}, \boldsymbol{\gamma}\right)=p+1\right\} \\
& =\sum_{p=0}^{P} \#\left\{\boldsymbol{\gamma} \in\left(\mathbf{H}_{q} \backslash \mathbf{S}_{N\left(2^{w-2}+1\right) / 2^{w}}\right): B_{2}\left(\mathbf{D}_{t}^{*}, \boldsymbol{\gamma}\right)=p+1\right\} \\
& -\sum_{p=0}^{P} \#\left\{\boldsymbol{\gamma} \in U\left(\mathbf{D}_{b}^{*}\right): B_{2}\left(\mathbf{D}_{t}^{*}, \gamma\right)=p+1\right\} \\
& =\#\left(\mathbf{H}_{q} \backslash \mathbf{S}_{N\left(2^{w-2}+1\right) / 2^{w}}\right)-\# U\left(\mathbf{D}_{b}^{*}\right), \\
& =2^{q}-\frac{N\left(2^{w-2}+1\right)}{2^{w}}-\# U\left(\mathbf{D}_{b}^{*}\right) \text {. } \\
& \sum_{p=0}^{P} \frac{{ }_{2}^{\#} C_{2}^{(p)}(\mathbf{D})}{(p+1)}=\sum_{p=0}^{b-2} \frac{{ }_{2}^{\#} C_{2}^{(p)}(\mathbf{D})}{(p+1)}+\frac{{ }_{2}^{\#} C_{2}^{(b-1)}(\mathbf{D})}{b} \\
& =2^{q}-\frac{N\left(2^{w-2}+1\right)}{2^{w}}-2^{k} .
\end{aligned}
$$

From (33) and (38), we have

By (36) and (37), it is obtained that 


$$
\begin{aligned}
\sum_{p=0}^{P} \frac{{ }_{2}^{\#} C_{2}^{(p)}\left(\mathbf{D}^{*}\right)}{(p+1)} & \geq \sum_{p=0}^{b-2} \frac{{ }_{2}^{\#} C_{2}^{(p)}\left(\mathbf{D}^{*}\right)}{(p+1)}+\frac{{ }_{2}^{\#} C_{2}^{(b-1)}\left(\mathbf{D}^{*}\right)}{b} \\
& >\sum_{p=0}^{b-2} \frac{2 C_{2}^{\#}(\mathbf{D})}{(p+1)}+\frac{{ }_{2}^{\#} C_{2}^{(b-1)}(\mathbf{D})}{b} .
\end{aligned}
$$

Then, according to (39)-(41), we obtain $\# U\left(\mathbf{D}_{b}^{*}\right)<2^{k}-1$ which contradicts Lemma 1 (i) and (ii).

For (b), similar to (a), when $k \leq q-w-1$, there are two different ways to choose $\mathbf{D}_{b}^{*}$ from $\mathbf{H}_{q}$ : (i) $\mathbf{D}_{b}^{*} \cap\{\mathbf{A}, \mathbf{B}, \mathbf{C}\}=\varnothing$ and (ii) $\mathbf{D}_{b}^{*} \cap\{\mathbf{A}, \mathbf{B}, \mathbf{C}\} \neq \varnothing$. With a similar argument to (a), case (i) results in $u_{1}=u_{2}=u_{3}=0$ while case (ii) results in $u_{1}>0, u_{2}>0$ or $u_{3}>0$. Therefore, if $\mathbf{D}^{*}$ is a $B^{2}$-GMC design, $\mathbf{D}_{b}^{*}$ should be chosen according to case (i), i.e., $\mathbf{D}_{b}^{*} \subset\left\{\mathbf{H}_{q-w},(\mathbf{q}-\mathbf{1}),(\mathbf{q}-\mathbf{1}) \mathbf{H}_{q-w}\right\}$. When choosing $\mathbf{D}_{b}^{*}$ according to (i), there should be $\mathbf{D}_{b}^{*} \cap\{\mathbf{q}-\mathbf{1}$, $\left.(\mathbf{q}-\mathbf{1}) \mathbf{H}_{q-w}\right\}=\varnothing$, i.e., $\quad \mathbf{D}_{b}^{*} \subset \mathbf{H}_{q-w} ;$ otherwise, $\mathbf{D}_{t}^{*} \cap U$ $\left(\mathbf{D}_{b}^{*}\right) \neq \varnothing$ noting that $\#\left\{U\left(\mathbf{D}_{b}^{*}\right) \cap\left(\mathbf{q}-\mathbf{1},(\mathbf{q}-\mathbf{1}) \mathbf{H}_{q-w}\right)\right\}$ $\geq 2^{k}>2^{r+1}-1$ as shown in Lemma 1 (iii). The remainder of the proof is similar to that of (a).

For (c), when $k>q-w-1$, there are two different ways to choose $\mathbf{D}_{b}^{*}$ from $\mathbf{H}_{q}$ : (i) $\mathbf{D}_{b}^{*} \cap\{\mathbf{B}, \mathbf{C}\}=\varnothing$ and (ii) $\mathbf{D}_{b}^{*} \cap\{\mathbf{B}, \mathbf{C}\} \neq \varnothing$. With a similar argument to (a), it is not hard to verify that case (ii) results in $u_{1}>0$ or $u_{2}>0$ while case (i) results in $u_{1}=u_{2}=0$. Therefore, if $\mathbf{D}^{*}$ is a $B^{2}$-GMC design, $\mathbf{D}_{b}^{*}$ should be an $s$-projection of $\mathbf{H}_{q-2}=\{\mathbf{B}, \mathbf{C}\}$. The remainder of the proof is similar to that of (a).

Remark 1. Theorem 2 shows that when constructing the $B^{2}$-GMC designs with $N / 4+1<n \leq 5 N / 16$, we can first partition the range $(N / 4+1,5 N / 16)$ into $q-4$ sequential subranges as $\left(N\left(2^{w-1}+1\right) / 2^{w+1}, N\left(2^{w-2}+1\right) / 2^{w}\right)$ with $w=$ $4,5, \ldots, q-1$ and then obtain the $B^{2}$-GMC designs according to Theorem 2 .

In the following, an example is provided to illustrate the construction method in Theorem 2 and Remark 1.

Example 2. Consider the constructions of $B^{2}$-GMC $2^{19-13}: 2^{s}$ designs for $s=1,3,4$. Here, $n=19, m=13, q=6$, and $N=64$. The values of the parameters $N$ and $n$ satisfy $N / 4+1<n \leq 5 N / 16$, i.e., $n \in(17,20]$. From Remark 1, we partition the range $(17,20]$ into two subranges $(17,18]$ because $w=5$ and $(18,20]$ because $w=4$. Since $19 \in(18,20]$, according to Theorem $2, \mathbf{D}_{t}$ should be the last 19 columns of $\mathrm{S}_{5 \mathrm{~N} / 16}$, where $\mathbf{S}_{5 N / 16}=D^{q-4}(\Phi(4))=\left(\mathbf{5}, \mathbf{5} \mathbf{H}_{2}, \mathbf{6}, \mathbf{6} \mathbf{H}_{2}, \mathbf{5 6}\left(\mathbf{H}_{4} \backslash \mathbf{H}_{2}\right)\right)$ from (18). Therefore, $\mathbf{D}_{t}=\left(\mathbf{5} \mathbf{H}_{2}, \mathbf{6}, \mathbf{6} \mathbf{H}_{2}, \mathbf{5 6}\left(\mathbf{H}_{4} \backslash \mathbf{H}_{2}\right)\right)$. Note that $5 N / 16-n=20-19=1$; then, $r=0$. Next, we choose $\mathbf{D}_{b}$ for $s=1,3,4$.

For $s=1$, we have $k=0$. According to Theorem 2 (a), $\mathbf{D}_{b}=\mathbf{5}$ as $\mathbf{H}_{0}=\varnothing$.

For $s=3$, we have $k=1$. According to Theorem $2(\mathrm{~b}), \mathbf{D}_{b}$ should be a 3-projection of $\mathbf{H}_{2}$, i.e., $\mathbf{D}_{b}=\mathbf{H}_{2}=(\mathbf{1}, \mathbf{2}, \mathbf{1 2})$.

For $s=4$, we have $k=2$. According to Theorem $2(\mathrm{c}), \mathbf{D}_{b}$ should be a 4-projection of $\mathbf{H}_{4}$. Without loss of generality, we choose $D_{b}=(1,2,12,3)$.

\section{Concluding Remarks}

The regular $2^{n-m}$ designs have wide applications in engineering, manufacturing industry, agriculture and medicine, and so on. When the size of experimental units is large, the inhomogeneity of experimental units results in unwanted variances of estimations of treatment effects. An essential way to solve this problem is to partition the experimental units into blocks.

There are two kinds of blocking problems as pointed out in [1]. One is called the single block variable problem which considers only a single block variable, and the other is called multi-block variable problem which considers two or more block variables. As stated in Section 1, experiments which involve multi-block variables are more widely concerned in practice than those which involve only a single block variable. However, due to the complexity of multi-block variable problem, the studies on constructing optimal designs with multi-block variables are relatively rare.

In this paper, we aim at exploring the theories and constructions of optimal blocked $2^{n-m}$ designs with multiblock variables. The prevalent $B^{2}$-GMC criterion is adopted. This criterion is preferable when there is some prior knowledge on the importance ordering of the treatment effects. The systematical construction methods of the $B^{2}$-GMC $2^{n-m}: 2^{s}$ designs with $N / 4+1 \leq n \leq 5 N / 16$ are developed. The construction methods are concise and easy to implement as indicated by the examples provided.

\section{Appendix}

Lemma A.1 is some result from Lemmas A.2, A.4, and A.5 in [21] and Lemma 1 in [25].

Lemma A.1. Suppose $\mathbf{O}$ is any s-projection of $\mathbf{H}_{q}$ with $2^{k} \leq s \leq 2^{k+1}-1$ and $k \leq q-2$.

(i) If $\mathbf{O} \cap\left\{\mathbf{q}, \mathbf{q} \mathbf{H}_{q-1}\right\}=\varnothing$, then $\#\left\{U(\mathbf{O}) \cap \mathbf{H}_{q-1}\right\}$ $\geq 2^{k+1}-1$, and when $\mathbf{O}$ has $k+1$ independent columns, the equality holds.

(ii) If $\mathbf{O} \cap\left\{\mathbf{q}, \mathbf{q} \mathbf{H}_{q-1}\right\} \neq \varnothing$, then $\#\left\{U(\mathbf{O}) \cap \mathbf{H}_{q-1}\right\}$ $\geq 2^{k}-1$, and when $\mathbf{O}$ has $k+1$ independent columns, the equality holds.

(iii) If $\mathbf{O} \cap\left\{\mathbf{q}, \mathbf{q} \mathbf{H}_{q-1}\right\} \neq \varnothing$, then $\#\left\{U(\mathbf{O}) \cap\left\{\mathbf{q}, \mathbf{q} \mathbf{H}_{q-1}\right\}\right\}$ $\geq 2^{k}$, and when $\mathbf{O}$ has $k+1$ independent columns, the equality holds.

(iv) If $\mathbf{O} \subset \mathbf{H}_{k+1}$, then $U(\mathbf{O})=\mathbf{H}_{k+1}$.

(v) If $\mathbf{O} \subset\left\{\mathbf{H}_{k}, \mathbf{q}, \mathbf{q} \mathbf{H}_{k}\right\}$, then $U(\mathbf{O})=\left\{\mathbf{H}_{k}, \mathbf{q}, \mathbf{q} \mathbf{H}_{k}\right\}$.

Lemma A.2 is some result of Lemma 1 in [34].

Lemma A.2. Suppose $\mathbf{O}$ consists of the last $n$ columns of $\left\{\mathbf{q}, \mathbf{q} \mathbf{H}_{q-1}\right\}$; then, $B_{2}\left(\mathbf{O}, \boldsymbol{\gamma}_{1}\right) \geq B_{2}\left(\mathbf{O}, \boldsymbol{\gamma}_{2}\right)$ if $\boldsymbol{\gamma}_{1}$ is ahead of $\boldsymbol{\gamma}_{2}$ in $\mathbf{H}_{q-1}$.

\section{Data Availability}

No data were used to support this study. 


\section{Conflicts of Interest}

The author declares that there are no conflicts of interest.

\section{Acknowledgments}

This study was supported by the National Natural Science Foundation of China (grant no. 11801331).

\section{References}

[1] S. Bisgaard, "A note on the definition of resolution for blocked 2 k-p designs," Technometrics, vol. 36, no. 3, pp. 308-311, 1994.

[2] R. R. Sitter, J. Chen, and M. Feder, "Fractional resolution and minimum aberration in blocked $2 \mathrm{n}-\mathrm{k}$ designs," Technometrics, vol. 39, no. 4, pp. 382-390, 1997.

[3] H. Chen and C. S. Cheng, "Theory of optimal blocking of $s^{n-m}$ designs," Annals of Statistics, vol. 27, no. 6, pp. 1948-1973, 1999.

[4] R. Zhang and D. Park, "Optimal blocking of two-level fractional factorial designs," Journal of Statistical Planning and Inference, vol. 91, no. 1, pp. 107-121, 2000.

[5] S. W. Cheng and C. F. J. Wu, "Choice of optimal blocking schemes in two-level and three-level designs," Technometrics, vol. 44, no. 3, pp. 269-277, 2002.

[6] H. Xu, "Blocked regular fractional factorial designs with minimum aberration," Annals of Statistics, vol. 8, no. 5, pp. 2534-2553, 2006.

[7] H. Xu and R. W. Mee, "Minimum aberration blocking schemes for 128-run designs," Journal of Statistical Planning and Inference, vol. 140, no. 11, pp. 3213-3229, 2010.

[8] S. Zhao, P. Li, and R. Karunamuni, "Blocked two-level regular factorial designs with weak minimum aberration," Biometrika, vol. 100, no. 1, pp. 249-253, 2013.

[9] S.-L. Zhao and P.-F. Li, "Construction of minimum aberration blocked two-level regular factorial designs," Communications in Statistics - Theory and Methods, vol. 45, no. 17, pp. 50285036, 2016.

[10] B.-J. Chen, P.-F. Li, M.-Q. Liu, and R.-C. Zhang, "Some results on blocked regular 2-level fractional factorial designs with clear effects," Journal of Statistical Planning and Inference, vol. 136, no. 12, pp. 4436-4449, 2006.

[11] S. Zhao, P. Li, and M.-Q. Liu, "On blocked resolution IV designs containing clear two-factor interactions," Journal of Complexity, vol. 29, no. 5, pp. 389-395, 2013.

[12] R. C. Zhang and R. Mukerjee, "General minimum lower-order confounding in block designs using complementary sets," Statistica Sinica, vol. 19, no. 4, pp. 1787-1802, 2009.

[13] S. Zhao, D. K. J. Lin, and P. Li, "A note on the construction of blocked two-level designs with general minimum lower order confounding," Journal of Statistical Planning and Inference, vol. 172, pp. 16-22, 2016.

[14] S.-L. Zhao and Q.-Q. Zhao, "Some results on constructing two-level block designs with general minimum lower order confounding," Communications in Statistics-Theory and Methods, vol. 47, no. 9, pp. 2227-2237, 2018.

[15] R. C. Zhang, P. Li, S. L. Zhao, and M. Y. Ai, "A general minimum lower-order confounding criterion for two-level regular designs," Statistica Sinica, vol. 18, no. 4, pp. 1689$1705,2008$.

[16] S. Zhao, P. Li, R. Zhang, and R. Karunamuni, "Construction of blocked two-level regular designs with general minimum lower order confounding," Journal of Statistical Planning and Inference, vol. 143, no. 6, pp. 1082-1090, 2013.

[17] Y. Zhao, S. Zhao, and M.-Q. Liu, "A theory on constructing blocked two-level designs with general minimum lower order confounding," Frontiers of Mathematics in China, vol. 11, no. 1, pp. 207-235, 2016.

[18] S.-L. Zhao and Q. Sun, "On constructing general minimum lower order confounding two-level block designs," Communications in Statistics-Theory and Methods, vol. 46, no. 3, pp. 1261-1274, 2017.

[19] J. Wei, P. Li, and R. Zhang, "Blocked two-level regular designs with general minimum lower order confounding," Journal of Statistical Theory and Practice, vol. 8, no. 1, pp. 46-65, 2014.

[20] C. F. J. Wu and M. S. Hamada, Experiments: Planning, Analysis and Optimization, Wiley, Hoboken, NJ, USA, 2 edition, 2009.

[21] Q. Zhao and S. Zhao, "Some results on two-level regular designs with multi block variables containing clear effects," Statistical Papers, vol. 60, no. 5, pp. 1569-1582, 2019.

[22] S. Zhao and Q. Zhao, "Minimum aberration blocked designs with multiple block variables," Metrika, vol. 84, no. 2, pp. 121-140, 2021.

[23] R. Zhang, P. Li, and J. Wei, "Optimal two-level regular designs with multi block variables," Journal of Statistical Theory and Practice, vol. 5, no. 1, pp. 161-178, 2011.

[24] Y. N. Zhao, S. L. Zhao, and M. Q. Liu, "On constructing optimal two-level designs with multi block variables," Journal of Systems Science and Complexity, vol. 31, pp. 1-14, 2018.

[25] Y. N. Zhao and S. L. Zhao, "Construction of optimal blocked designs with multi block variables," AIMS mathematics, vol. 6, no. 6, pp. 6293-6308, 2021.

[26] B. Tang and C. F. J. Wu, "Characterization of minimum aberration $2^{n-k}$ designs in terms of their complementary designs," Annals of Statistics, vol. 25, no. 6, pp. 2549-2559, 1996.

[27] C. F. J. Wu and Y. Chen, "A graph-aided method for planning two-level experiments when certain interactions are important," Technometrics, vol. 34, no. 2, pp. 162-175, 1992.

[28] G. E. P. Box and J. S. Hunter, "The $2^{k-p}$ fractional factorial designs I and II," Technometrics, vol. 3, no. 4, pp. 311-351, 1961.

[29] Y. Cheng and R. Zhang, "On construction of general minimum lower order confounding $2 \mathrm{n}-\mathrm{m}$ designs with , $(N / 4)+$ $1 \leq n \leq 9 N / 32$," Journal of Statistical Planning and Inference, vol. 140, no. 9, pp. 2384-2394, 2010.

[30] H. Wu and C. F. J. Wu, "Clear two-factor interactions and minimum aberration," Annals of Statistics, vol. 30, no. 5, pp. 1496-1511, 2002.

[31] R. M. Block and R. W. Mee, "Second order saturated resolution IV designs," Journal of Statistical Theory and Applications, vol. 2, no. 2, pp. 96-112, 2003.

[32] A. A. Davydov and L. M. Tombak, "Quasi-perfect linear binary codes with distance 4 and complete caps in projective geometry," Problems of Information Transmission, vol. 25, no. 4, pp. 265-275, 1990.

[33] R. Zhang and Y. Cheng, "General minimum lower order confounding designs: an overview and a construction theory," Journal of Statistical Planning and Inference, vol. 140, no. 7, pp. 1719-1730, 2010.

[34] Q. Zhou, N. Balakrishnan, and R. Zhang, "The factor aliased effect number pattern and its application in experimental planning," Canadian Journal of Statistics, vol. 41, no. 3, pp. 540-555, 2013. 